\title{
Spontaneous bilateral adrenal hemorrhage of pregnancy
}

\author{
Kavya Jonnalagadda ${ }^{1 *}$, Nisha Bhavani ${ }^{1}$, Praveen V. Pavithran ${ }^{1}$, \\ Harish Kumar ${ }^{1}$, Usha V. Menon ${ }^{1}$, Chithra R. ${ }^{2}$
}

\begin{abstract}
${ }^{1}$ Department of Endocrinology, Amrita Institute of Medical Sciences, Amrita University, Kochi, India
${ }^{2}$ Department of Obstetrics and Gynaecology, Amrita Institute of Medical Sciences, Amrita University, Kochi, India
\end{abstract}

Received: 12 December 2016

Accepted: 06 January 2017

\section{*Correspondence:}

Dr. Kavya Jonnalagadda,

E-mail: jdkavya@gmail.com

Copyright: ( ) the author(s), publisher and licensee Medip Academy. This is an open-access article distributed under the terms of the Creative Commons Attribution Non-Commercial License, which permits unrestricted non-commercial use, distribution, and reproduction in any medium, provided the original work is properly cited.

\begin{abstract}
Spontaneous adrenal hemorrhage of pregnancy is an acute hemorrhage into the adrenal gland in pregnancy in the absence of trauma, tumor or decoagulant therapy. This can have catastrophic consequences on the mother and the baby and if the hemorrhage involves both the adrenal glands the risk is aggravated because of the high incidence of resulting adrenal insufficiency. We report a case of spontaneous bilateral adrenal hemorrhage in pregnancy resulting in adrenal crisis. A 26 year old primigravida presented at 32 weeks of gestation initially with right sided infrascapular pain and one month later with similar pain in the left side associated with high blood pressure. Imaging with ultrasound and MRI was suggestive of bilateral adrenal mass probably hemorrhage; 2 days following the second episode of pain she developed drowsiness and hypotension and a diagnosis of primary adrenal insufficiency was confirmed by a low serum cortisol and high ACTH. She stabilized with hydrocortisone therapy and the fetus was closely monitored. At 37 weeks she had a normal vaginal delivery under steroid cover. Repeat MRI abdomen 3 months after delivery showed resolution of the hemorrhage but biochemically she continued to be cortisol insufficient at 1 year of follow up. Prompt diagnosis of adrenal hemorrhage in pregnancy and treatment of adrenal insufficiency along with close fetal monitoring usually results in good perinatal outcome in spontaneous adrenal hemorrhage of pregnancy.
\end{abstract}

Keywords: Adrenal, Cortisol, Hemorrhage, Pregnancy

\section{INTRODUCTION}

Spontaneous adrenal hemorrhage is the hemorrhage in to the adrenals in the absence of preceding trauma, decoagulant therapy and tumor. ${ }^{1}$ Unless diagnosed and managed promptly it can have disastrous consequences on the mother and the baby.

\section{CASE REPORT}

A 26 year old primigravida presented at 32 weeks of gestation with left infrascapular pain radiating to left side of the abdomen associated with vomiting, palpitation, low grade fever and high blood pressure. Patient had similar complaints on right side one month back.
Ultrasound abdomen done at that time showed a right adrenal mass. A work up for pheochromocytoma and Cushing's was done which were reported to be normal. She was diagnosed with subclinical hypothyroidism in the first trimester and was on levothyroxine replacement.

At the time of presentation she was hemodynamically stable, her heart rate was 100/minute and blood pressure was $150 / 100 \mathrm{~mm}$ of $\mathrm{Hg}$. Fundal height was 32 weeks and fetal heart rate was 130/minute. An MRI of the abdomen was done which showed an isointense lesion of $4 \times 5 \mathrm{~cm}$ on the left adrenal and hyperintense lesion of around $3 \times 4 \mathrm{~cm}$ on the right adrenal gland suggestive of acute and subcaute bleed respectively (Figures 1 and 2). 


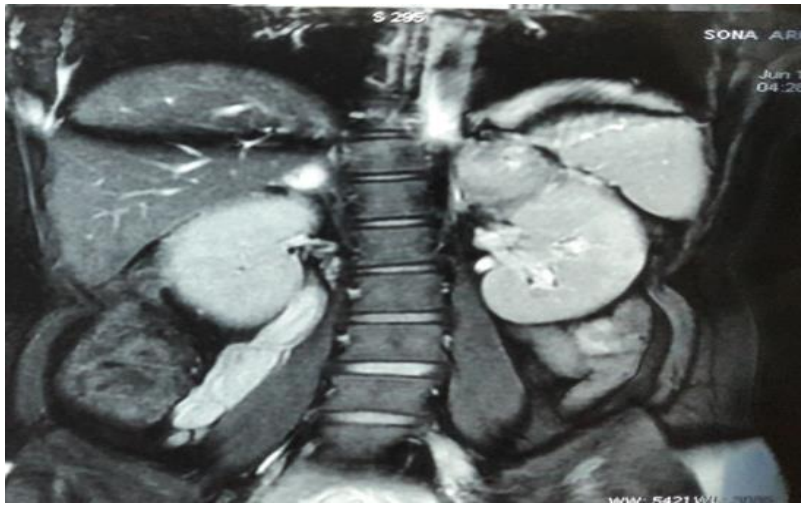

Figure 1: Coronal T2 image of MRI abdomen showing acute hemorrhage on left side and subacute

hemorrhage on right side. Acute hemorrhage $<7$ days appears hypointense and $>1-7$ weeks appear hyperinense on $\mathrm{T} 2$.

Biochemistry revealed normal hemogram, liver and renal function tests and electrolytes. She had urinary tract infection with significant growth of Ecoli and was started on culture specific antibiotics. Since the hypertension was persisting she was started on nifedipine. Hormonal work up for pheochromocytoma was done with 24 hour urine fractionated metanephrines which came back as normal. Cortisol axis was not assessed initially since she had received one dose of betamethasone from the referring hospital.

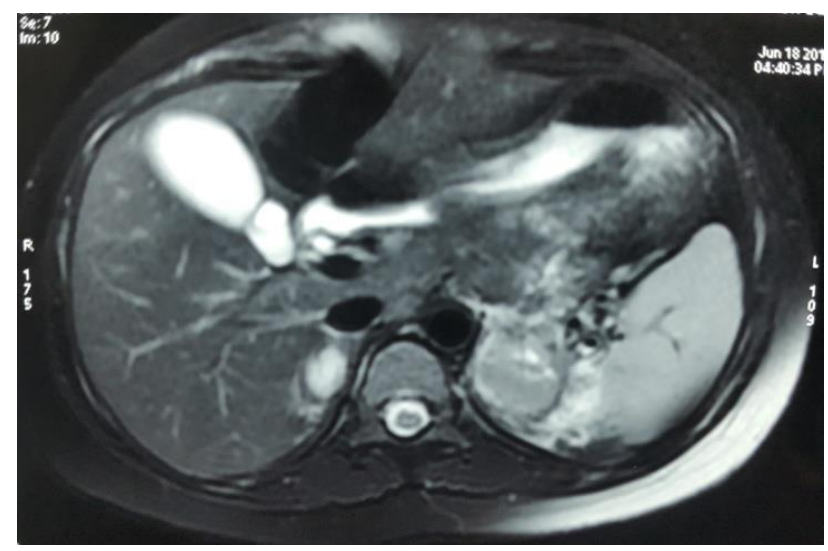

Figure 2: Axial T2 image of MRI abdomen showing acute hemorrhage on left side and subacute hemorrhage on right side.

On the $3^{\text {rd }}$ day of hospitalization she had increasing tiredness, drowsiness and increased frequency of vomiting with associated hypotension with hyponatremia. On the clinical suspicion of adrenal crisis samples were collected for random cortisol, ACTH and stimulated cortisol and the patient was immediately started on Injection Hydrocortisone $50 \mathrm{mg}$ IV Q8hourly with which her symptoms dramatically improved. Random cortisol was $6.2 \mathrm{ug} / \mathrm{dl}$, ACTH was $216.9 \mathrm{pg} / \mathrm{ml}$ and the stimulated cortisol was $6.0 \mathrm{ug} / \mathrm{dl}$ and $6.2 \mathrm{ug} / \mathrm{dl}$ at 60 and 90 minutes. A diagnosis of primary adrenal insufficiency was confirmed. Work up for secondary causes of hemorrhage was done. Her coagulation profile was normal. Anti phospholipid antibody IgG and IgM were 1.47 units $/ \mathrm{ml}$ and 1.14units/ml (Normal 0-9.9) and was negative. She was closely monitored with daily blood pressure, serum electrolytes and fetal monitoring and gradually her hydrocortisone dose was tapered to oral tablet $20 \mathrm{mg}$ 10mg-5 $\mathrm{mg}$ and she was discharged

At 37 weeks patient was readmitted for safe confinement. Under parenteral steroid cover, PGE1 induction was done and labour was augmented with ARM and oxytocin and she delivered by vaginal route a live male baby weighing $2.7 \mathrm{~kg}$. Following labour she was continued on oral hydrocortisone $20 \mathrm{mg}$ three times daily and gradually tapered to $10-5-5 \mathrm{mg}$.
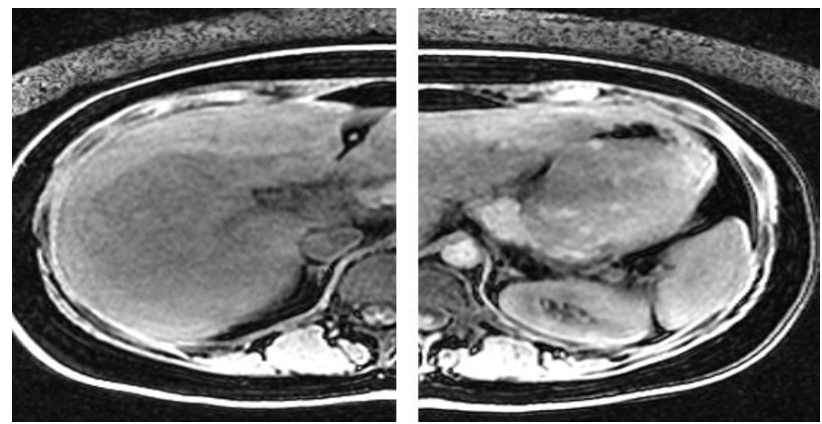

Figure 3: Repeat MRI abdomen done 3 months postdelivery.

USG abdomen and pelvis was done post-delivery to look for adrenal status which was suggestive of bilateral suprarenal hypoechoic lesions of $2 \times 1.5 \mathrm{~cm}$ and $3 \times 4 \times 5.2$ $\mathrm{cm}$ in the right and left adrenals. MRI abdomen done 3 months after delivery showed complete resolution of the hemorrhage and a normal right adrenal and bulky left adrenal (Figure 3). Patient is on follow up since then and at 1 year post-delivery she is continuing to need hydrocortisone replacement since her basal and stimulated cortisol are still 3.3ug/dl and 3.6ug/dl respectively.

\section{DISCUSSION}

Spontaneous adrenal hemorrhage is the hemorrhage into the adrenal glands in the absence of preceding trauma, decoagulant therapy and tumor. ${ }^{1}$ It's usually unilateral when right adrenal is more affected than left and rarely bilateral. Its incidence in pregnancy is not known and is extremely rare, with a prevalence of $0.14-1.1 \%$ in postmortem studies. $^{2}$

There are different theories implicated in adrenal hemorrhage of pregnancy. Adrenal gland has a rich arterial supply in the subcapsular plexus with limited venous drainage into delicate medullary sinusoids through few branches in the corticomedullary junction .In hypotension, medullary sinusoids are at risk of ischemic necrosis and when normal arterial pressure is restored, 
reperfusion hemorrhage occurs. Adrenocortical hyperplasia and hypertrophy occurring in pregnancy. The high ACTH increases the arterial blood supply of adrenals. Adrenal vein spasm induced by high catecholamine secretion might lead to venous congestion and hemorrhage into the adrenals in pregnancy. Adrenal vein thrombosis induced by coagulopathies like sepsis, antiphospholipid antibody syndrome, disseminated intravascular coagulation and heparin-induced thrombocytopenia, may also lead to venous stasis and hemorrhage. ${ }^{3,5-7}$ Other risk factors for adrenal hemorrhage in pregnancy are spontaneous abortion, toxemia of pregnancy, post-partum hemorrhage and torsion of an ovarian cyst.

Primary symptoms of adrenal hemorrhage include pain in the abdomen, chest or back, vomiting, and rarely fever. Acute abdomen with guarding, rigidity and rebound tenderness is seen in $15 \%-20 \%$ cases. Hypotension is rare occurring in $15 \%$ of cases. ${ }^{2}$ Anemia and prolonged INR may occur in few cases. Clinical differential diagnosis includes abruption placentae, ectopic pregnancy, uterine rupture and many other non pregnancy related causes (pancreatitis, appendicitis, diverticulitis nephrolithiasis etc). Unilateral adrenal hemorrhage usually does not manifest with adrenal insufficiency but patients with bilateral adrenal hemorrhage may present with acute adrenal crisis which occurs only when $90 \%$ of the adrenal cortex is destroyed. ${ }^{2}$

The diagnosis of adrenal insufficiency in pregnancy is also challenging because of the aberrations in steroidogenesis occuring in normal pregnancy .There is a markedly increased maternal and placental ACTH and cortisol synthesis and very high cortisol binding globulin levels resulting in high serum cortisol and ACTH levels in pregnancy. The diagnostic cut offs for adrenal insufficiency are not well validated in pregnancy An early morning cortisol $<3 \mathrm{ug} / \mathrm{dl}$ in the nonstressed state associated with typical symptoms confirms the diagnosis and a basal or stimulated cortisol value more than 30 $\mathrm{mcg} / \mathrm{dl}$ usually rules out adrenal insufficiency in pregnancy. .8

Imaging is the key diagnostic modality for adrenal hemorrhage in pregnancy. Ultrasonogram (USG) will be the initial imaging choice followed by MRI without contrast. Acute hemorrhage on USG appears hyperintense and chronic hemorrhage appears hypointense. MRI abdomen is more specific and can predict the age of hemorrhage. Acute hemorrhage appears iso to hypointense on $\mathrm{T} 1$ and markedly hypointense in $\mathrm{T} 2$ sequences.Subacute hemorrhage appears hyperintense in both T1 and T2 sequences. Chronic hemorrhage has a hypointense rim with hyperintense core on $\mathrm{T} 1$ and $\mathrm{T} 2$ sequences. ${ }^{10}$ Other adrenal masses like adrenal adenoma, pheochromocytoma, granulomatous disease of adrenals will be the probable differential diagnoses for adrenal hemorrhage in MRI. An underlying mass can be ruled out only by contrast MRI. Secondary causes for hemorrhage should be ruled out like disseminated intravascular coagulation, thrombotic thrombocytopenic purpura, antithrombin III deficiency, protein $\mathrm{C}$ deficiency, protein $\mathrm{S}$ deficiency, factor $\mathrm{V}$ Leiden mutation, prothrombin gene mutation G20210A, and antiphospholipid antibody syndrome

Management of SAH varies from patient to patient. Conservative management will suffice in most of the cases except in those with massive retroperitoneal hemorrhage, refractory hemorrhagic shock, or evident clinical deterioration of maternal and fetal conditions. Emergency adrenalectomy or angioembolisation can be done to control bleeding and preterm delivery induced when there is eclampsia with uncontrolled adrenal hemorrhage.

Mode of delivery in pregnancy complicated by SAH is not well established given the fact that the stress of labour can cause further hemorrhage. But vaginal delivery can be undertaken in a stable patient. Stress dose of steroids should be given during labour if there is adrenal insufficiency. Risk of recurrence in next pregnancy is not very clear. Majority of cases reported in literature showed recovery of axis in around 6 months time. Few like ours had prolonged adrenal insufficiency.

\section{CONCLUSION}

Adrenal hemorrhage should be considered as a differential in pregnant women presenting with acute abdomen. Prompt diagnosis and treatment usually lowers the maternal and fetal mortality and morbidity.

\section{Funding: No funding sources \\ Conflict of interest: None declared \\ Ethical approval: Not required}

\section{REFERENCES}

1. Wani MS, Naikoo ZA, Malik MA. Spontaneous adrenal hemorrhage during pregnancy: review of literature and case report of successful conservative management. Journal of the Turkish German Gynecology Association. 2011;12(4):263-5.

2. Gavrilova-Jordan L, Edmister WB, Farrell MA, Watson WJ. Spontaneous adrenal hemorrhage during pregnancy: a Review of literature and case report of successful conservative management. Obstet Gynecol Surv. 2005;60:191-5.

3. Kovacs KA, Lam YM, Pater JL. Bilateral massive adrenal hemorrhage. Assessment of putative risk factors by case controlled method. Medicine (Baltimore). 2001;80:45-53.

4. Imag NN, Tutuncu Y, Tuna MM, Dogan BA, Berker D. Idiopathic spontaneous adrenal hemorrhage in the third trimester of pregnancy. Case reports in Medicine. 2013;Article ID 912494, 2 pages.

5. Ramon I, Mathian A, Bachelot A, Hervier B, Haroche J, Boutin-Le Thi Huong D, et al. Primary 
Adrenal Insufficiency Due to Bilateral Adrenal Hemorrhage-Adrenal Infarction in the Antiphospholipid Syndrome: Long-Term Outcome of 16 Patients. J Clin Endocrinol Metab. 2013;98(8):3179-89.

6. Adem PV, Montgomery CP, Husain AN. Staphylococcus aureus sepsis and the WaterhouseFriderichsen syndrome in children. N Engl J Med. 2005;353(12):1245-51.

7. Ketha S, Smithedajkul P, Vella A, Pruthi R, Wysokinski W, McBane R. Adrenal haemorrhage due to heparin-induced thrombocytopenia. Thromb Haemost. 2013;109(4):669-75.

8. Suri D, Moran J, Hibbard JU. Assessment of adrenal reserve in pregnancy: Defining the normal response to adrenocorticotrophin stimulation test. J Clin Endocrinol Metab. 2006;91(10):3866-72.

9. Lindsay JR, Nieman KL. The HypothalamicPituitary-Adrenal Axis in Pregnancy: Challenges in Disease Detection and Treatment. Endocrine Reviews. 2005;26(6):775-99.

10. Taffel M, Haji-Momenian S, Nikolaidis P, Miller FH. Adrenal imaging: a comprehensive review. Radiol Clin North Am. 2012;50(2):219-43.

Cite this article as: Jonnalagadda K, Bhavani N, Pavithran PV, Kumar H, Menon UV, Chithra R. Spontaneous bilateral adrenal hemorrhage of pregnancy. Int J Reprod Contracept Obstet Gynecol 2017;6:772-5. 